\title{
FOUR-DIMENSIONAL DATA ASSIMILATION FOR COUPLED PHYSICAL-ACOUSTICAL FIELDS
}

\author{
P.F.J. LERMUSIAUX ${ }^{1}$ AND C.S. CHIU ${ }^{2}$ \\ ${ }^{1}$ Harvard University, DEAS, Pierce Hall G2A, 29 Oxford Street, Cambridge MA 02318, USA \\ E-mail: pierrel@pacific.harvard.edu \\ ${ }^{2}$ Naval Postgraduate School, Monterey, CA 93943, USA \\ E-mail: chiu@nps.navy.mil
}

\begin{abstract}
The estimation of oceanic environmental and acoustical fields is considered as a single coupled data assimilation problem. The four-dimensional data assimilation methodology employed is Error Subspace Statistical Estimation. Environmental fields and their dominant uncertainties are predicted by an ocean dynamical model and transferred to acoustical fields and uncertainties by an acoustic propagation model. The resulting coupled dominant uncertainties define the error subspace. The available physical and acoustical data are then assimilated into the predicted fields in accord with the error subspace and all data uncertainties. The criterion for data assimilation is presently to correct the predicted fields such that the total error variance in the error subspace is minimized. The approach is exemplified for the New England continental shelfbreak region, using data collected during the 1996 Shelfbreak Primer Experiment. The methodology is discussed, computational issues are outlined and the assimilation of model-simulated acoustical data is carried out. Results are encouraging and provide some insights into the dominant variability and uncertainty properties of acoustical fields.
\end{abstract}

\section{Introduction}

Ocean acousticians are mainly interested in the distribution and composition of sound pressure fields in the ocean. Physical oceanographers are mainly interested in the oceanic motions and physical properties of the fluid ocean. In both disciplines, the estimation of the variables of interest is challenging because oceanic variability occurs on multiple interactive scales and is difficult to observe. To our knowledge, even though both disciplines employ sophisticated techniques for the estimation of their respective variables, few studies have envisioned a truly coupled four-dimensional estimation, including both the acoustic and oceanic variables in the state vector.

Ocean acoustic wavefields depend on the three-dimensional sound speed field whose evolution is a function of the fluid ocean physics (temperature, salinity, ambient pressure, etc.) and bottom attributes (reflectivity, attenuation, etc.). Due to these dynamical couplings, a joint estimation of acoustical-physical fields is attractive. First, sound waves propagate over long distances in the ocean and acoustic measurements can thus provide valuable integrated oceanic data for physical studies. Similarly, by natural variability, spatial and temporal correlations among environmental properties occur on multiple scales and even sparse measurements of this variability thus provide valuable information for acoustical studies. In fact, accurate physical inputs are necessary for successful acoustic 
simulations. Finally, even without natural data, acoustical and physical models are sources of coupled data which can be shared to improve estimates.

Considering uncertainties, the mathematical equations used to describe the environment and acoustic properties are approximate, as well as their analytical or numerical solutions. The natural physical and acoustical data are limited in accuracy and coverage. Because of these uncertainties and because of the above dynamical couplings, carrying out a joint estimation is likely to provide substantial advantages. In such an estimation, the sources of information, environmental and acoustical data, and ocean dynamics and sound propagation models, are combined by data assimilation. This combination is optimal in the sense that each information is weighted in accord with its uncertainty. In principle, this process provides better estimates of parameters and properties than can be obtained by using only the observations or models alone. The acoustical data improve physical fields; the physical data improve acoustical fields. Of course, should optimal estimates fail to be accurate, a priori assumptions about uncertainties are revised, and models and data sets improved. This manuscript outlines an approach for such four-dimensional (time and space) physical-acoustical estimations via coupled data assimilation and carries out an illustrative example based on data and simulations for the New England continental shelfbreak region.

\section{Methodology}

Data assimilation [11] combines dynamical models and data sets by quantitative minimization of a criterion or cost function. The links between observational data and dynamical model fields and parameters are provided by measurement models. Since dynamical models, data sets and measurement models are all approximate, they all involve an error component, i.e. the error models. These error models are here stochastic. The dynamical models, data sets and measurement models, and data assimilation scheme are now described.

\subsection{Coupled dynamical models}

Ocean Physics Model. The physical state variables are temperature $T$, salinity $S$, velocity $\mathbf{u}$ and pressure $p_{w}$. For this study, their mesoscale evolution is computed by the PrimitiveEquation model (Eqs. 1-7) of the numerical Harvard Ocean Prediction System, e.g. [10]. Atmospheric fluxes based on surface buoy time-series are imposed at the surface. Model parameters and boundary conditions were calibrated based on data and sensitivity studies.

$$
\begin{array}{ll}
\text { Momentum } & \rho \frac{D \mathbf{u}}{D t}+2 \rho \Omega \wedge \mathbf{u}=-\nabla p_{w}+\nabla \cdot \tau^{v}+\rho \mathbf{g} \\
\text { Thermal energy } & \rho C_{p} \frac{D T}{D t}=\nabla \cdot(k \nabla T)+\rho Q \\
\text { Cons. of salt } & \rho \frac{D S}{D t}=\nabla \cdot\left(k^{s} \nabla S\right)+\rho Q^{S} \\
\text { Cons. of mass } & \nabla \cdot \mathbf{u}=0 \\
\text { Eqn. of state } & \rho(\mathbf{r}, z, t)=\rho\left(T, S, p_{w}\right) \\
\text { Sound speed eqn. } & c(\mathbf{r}, z, t)=C\left(T, S, p_{w}\right) \\
\text { Wave eqn. } & \nabla^{2} p_{s}(\mathbf{r}, z, t)=\frac{1}{c(\mathbf{r}, z, t)} \frac{\partial^{2} p_{s}(\mathbf{r}, z, t)}{\partial t^{2}}
\end{array}
$$

Acoustic Model. The acoustic coupled normal mode model $[1,2,3]$ solves a linearized wave equation (Eq. 9) governing sound pressure $p_{s}$ whose water-column parameter is the 


\section{COUPLED PHYSICAL-ACOUSTICAL DATA ASSIMILATION}

4D sound-speed field $c$ (Eq. 8). The acoustic pressure is decomposed in the frequency domain into slowly-varying complex envelopes that modulate (mode by mode) analytic, rapidly-varying, adiabatic-mode solutions. Given sound speed, density, attenuation rate and bathymetry vertical cross-sections, the acoustic state is obtained by integrating differential equations governing the complex modal envelopes. Model output contains sound pressure, transmission loss, and travel time, phase and amplitude of the individual modes.

With model errors, a stochastic extension of Eqs. 1-9 is solved. Presently, only physical model errors are employed: they represent uncertainties due to sub-mesoscales and internal tides not accounted for in the deterministic mesoscale simulations (Eqs. 1-7).

\subsection{Data sets and measurement models}

Presently, in situ physical data include profiles of temperature, salinity and velocities. Remotely-sensed data include satellite data (SSH, SST). Before being utilized, the raw measurements from XBTs, CTDs, ADCPs, current meters and satellites are processed via averaging, filtering, de-aliasing and calibration. Acoustic sensor observations are also processed to lead acoustic data such as sound pressure, travel time and transmission loss (TL). These coupled data are linked to the dynamics (Eqs. 1-9) by measurement models. Note that in general such models can be sophisticated so as to efficiently link the non-observed state variables in Eqs. (1-9) to the observed data and so as to account for all uncertainties, including these that occur in the processing.

\subsection{Data assimilation approaches: discrete equations and computations}

In discrete terms, the physical-acoustical state is represented by a coupled state vector, $\mathbf{x}$, which is evolved from $\mathbf{x}\left(t_{0}\right)=\widehat{\mathbf{x}}_{0}$ based on, $d \mathbf{x}=\mathcal{M}(\mathbf{x}) d t+d \boldsymbol{\eta}$, where $\mathcal{M}$ is the coupled model operator and $d \boldsymbol{\eta}$ are stochastic uncertainties. At time $t_{k}$, measurement models are of the form, $\mathbf{y}_{k}=\mathcal{H}\left(\mathbf{x}_{k}\right)+\epsilon_{k}$, where $\mathbf{y}_{k}$ is the observed data, $\mathcal{H}$ the measurement model operator and $\epsilon_{k}$ the stochastic uncertainties. The goal of the present four-dimensional data assimilation is to minimize the trace of the a posteriori error covariance of the coupled state, $\mathbf{P}_{k}^{p}(+)$, i.e. find $\mathbf{x}_{k}$ such that $J_{k}=\operatorname{tr}\left[\mathbf{P}_{k}^{p}(+)\right]$ is minimized using $\left[\mathbf{y}_{0}, \ldots, \mathbf{y}_{k}\right]$.

One, several or all of the acoustic variables can be included in the joint ocean-acoustic state space. Similarly, oceanic and acoustic fields can each be defined on both the physical and acoustical grids. This importantly extends the approach where acoustic computations are restricted to a high-resolution vertical plane while ocean computations are restricted to a lower-resolution volume grid. Solving for the physics and acoustics on both grids by data assimilation then provides internal wave physical resolution along the acoustic paths and range-averaged acoustical resolution on the whole ocean volume. Even though this is an ultimate goal, presently, our new coupled estimation is only illustrated for the ocean and acoustic states on their respective grids.

\subsection{Data assimilation scheme}

The coupled data assimilation methodology for field and uncertainty estimations is Error Subspace Statistical Estimation $[4,5,6]$. ESSE is based on evolving an error subspace, of variable size, that spans and tracks the scales and processes where dominant errors occur. With ESSE, the sub-optimal reduction of errors is itself optimal. Presently (Fig. 1), the 


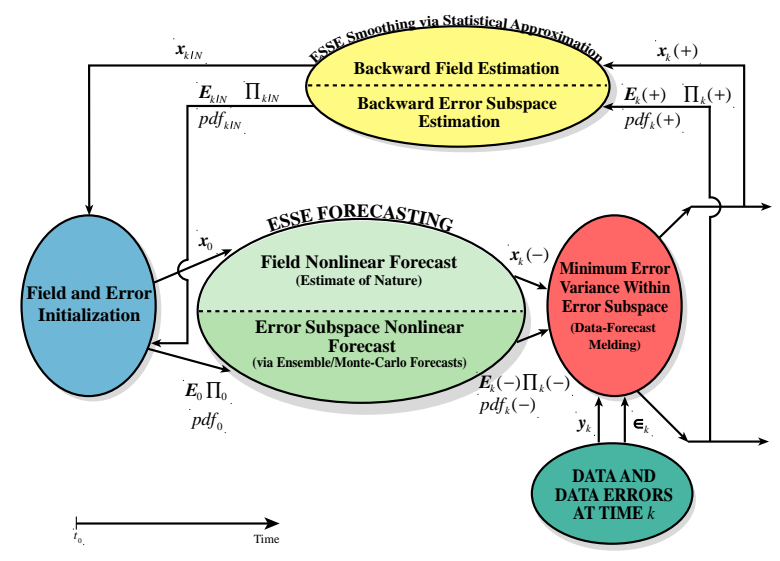

Figure 1. Five main components of the present ESSE system.

error subspace is initialized by decomposition on multiple scales [7] and the resulting estimate of the initial error eigendecomposition or error pdf (Fig. 1, blue oval) is used to perturb the initial state $\mathbf{x}_{0}$. To evolve the physical fields and uncertainties (Fig. 1, light green oval), an ensemble of stochastic ocean model integrations (Eqs. 1-7) are carried out in parallel. The ensemble size is controlled by convergence criteria; when satisfied, the ensemble of ocean states leads to the physical forecast of nature $\mathbf{x}_{k}(-)$ and to its error estimate, e.g. the error eigenvectors $\mathbf{E}_{k}(-)$ and eigenvalues $\boldsymbol{\Pi}_{k}(-)$ obtained by normalized SVD. With these physical fields and uncertainties, one computes an ensemble of 3D sound-speed fields. Each sound-speed realization then enters as a 3D parameter (Eq. 8) in an integration of the acoustic propagation model (Eq. 9). The acoustical ensemble is computed and, as for the physics, its size is controlled by convergence criteria. When satisfied, the acoustical and physical ensembles are concatenated to provide the coupled predicted fields and uncertainties. At this stage, the data and their error estimates (Fig. 1, dark green oval) are employed. Data-forecast misfits are computed and used to correct the predicted fields by minimum error variance estimation in the predicted physical-acoustical error subspace (Fig. 1, red oval). During this melding, acoustical data influence the physical state and vice-versa. The outputs are the a posteriori coupled fields $\mathbf{x}_{k}(+)$ and a posteriori coupled errors, e.g. $\mathbf{E}_{k}(+), \boldsymbol{\Pi}_{k}(+)$. A posteriori data misfits are then calculated and used for adaptive learning of the dominant errors, e.g. [5]. This learning of errors from misfits can be necessary because error estimates are themselves uncertain. Ultimately, the smoothing via ESSE [6] can be carried out to correct, based on the data at times $t_{k}$, the initial coupled fields and uncertainties at $t_{0}$ : this leads to $\mathbf{x}_{0 / N}$ and e.g., $\mathbf{E}_{0 / N}, \boldsymbol{\Pi}_{0 / N}$.

\section{Illustrative example}

The physics considered are the mesoscale dynamics of the Middle Atlantic Bight shelfbreak front, including remote influences from the shelf, slope and deep ocean. The acoustics is the transmission of low-frequency sound from the continental slope, through the shelfbreak front, onto the shelf. These dynamics, and also the model parameters, data assimilated in the physical model, and acoustical-physical uncertainties are described in [8].

The coupled assimilation via ESSE is illustrated for the 3D physical fields and 2D trans- 


\section{COUPLED PHYSICAL-ACOUSTICAL DATA ASSIMILATION}

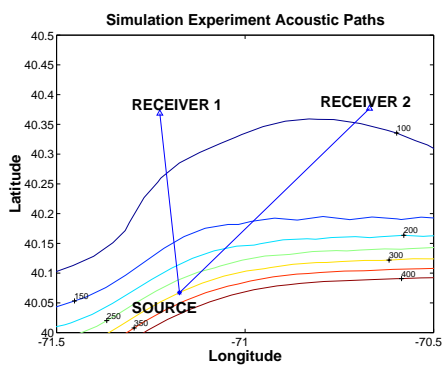

Figure 2. Acoustic paths considered (as in Shelfbreak-PRIMER), overlaid on bathymetry.

mission loss along an actual Shelfbreak-PRIMER [9] acoustic path (Fig. 2). The $224 \mathrm{~Hz}$ source is at $300 \mathrm{~m}$ depth. The acoustical data assimilated are simulated towed-array TL data along path 1, i.e. TL1 (the assimilation of simulated VLA's data at receiver 1 was also successful but is not shown). These model data were extracted from a physical-acoustical realization that is independent from the ensemble of 79 simulations carried out during the ESSE computations (Sect. 2.4). This independent realization is called the "true" ocean and such an assimilation exercise is called an "identical twin experiment". Goals in such an experiment are to study the assimilation in an ideal situation and to find out if the a posteriori fields become close to the known "true" fields. Presently, TL observations are made at constant $70 \mathrm{~m}$ depth, every $50 \mathrm{~m}$ from $r=150 \mathrm{~m}$ to almost receiver 1 . These are very sub-sampled data since the $(r, z)$ grid resolution is $5 \mathrm{~m}$ by $5 \mathrm{~m}$.
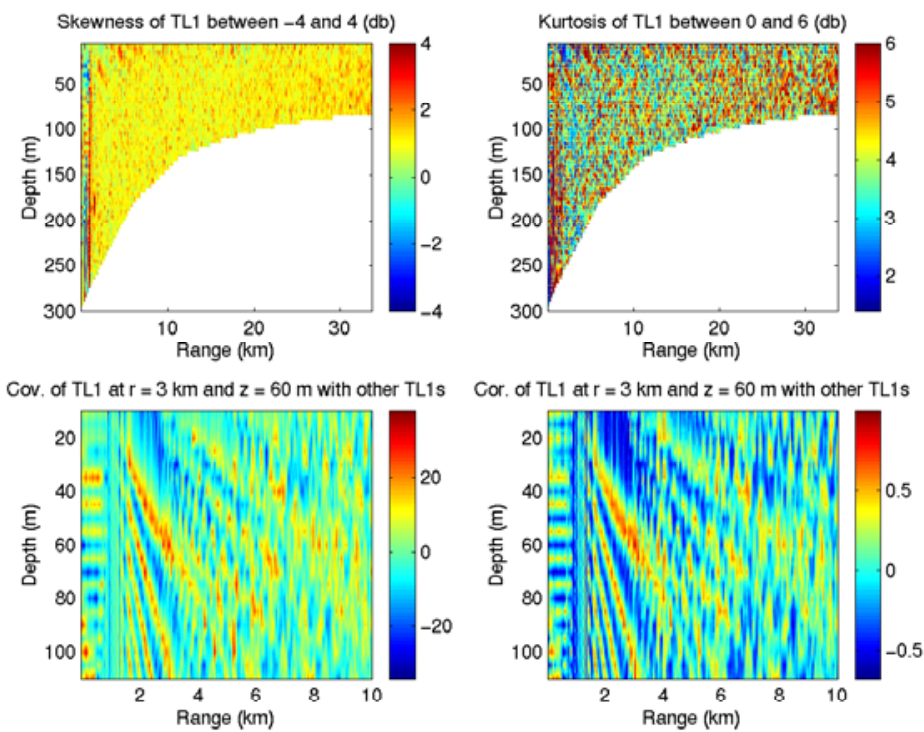

Figure 3. TL error statistics: sample skewness, kurtosis and zoom on a covariance/correlation field.

Figures 3 and 4 illustrate the predicted (a priori) TL uncertainties computed by ESSE. Except in the near field (where numerical errors in the acoustical model, Sect. 2.1, are the largest), the skewness and kurtosis (Figs. 3a-b) of the error pdf are patchy on small scales but relatively uniform at larger scales (around 1 and 5, respectively). The sensitivities 
of this pdf result to the size of the ensemble and to the properties of physical error pdf estimates need to be investigated. A sample estimate of error covariance and correlation functions between TL at ( $r=3 \mathrm{~km}, z=6 \mathrm{~m}$ ) with other TLs (Figs. 3c-d) clearly shows the influence of acoustic wave patterns as they propagate through the shelfbreak front.

Figure 4 shows the four dominant singular vectors of the $79 \mathrm{TL}$ deviations from the mean (a priori) TL. These ESSE estimates of the non-dimensional error eigenvectors account for 12.4, 6.0, 4.2 and 3.6 percent of the $2 \mathrm{D}$ acoustic error variance, respectively. They indicate the directions of the acoustic state space with the largest uncertainty. Presently, since we are just before the first assimilation of acoustic data, they also relate to the dominant acoustic variability. The first vector is linked to sub-thermocline propagation of sound onto the shelf, the second to uncertainties at the front due to the locally higher physical variability and the third and fourth (eigenvectors of similar eigenvalues) to successive reflections of sound waves between the thermocline and the bottom/surface.
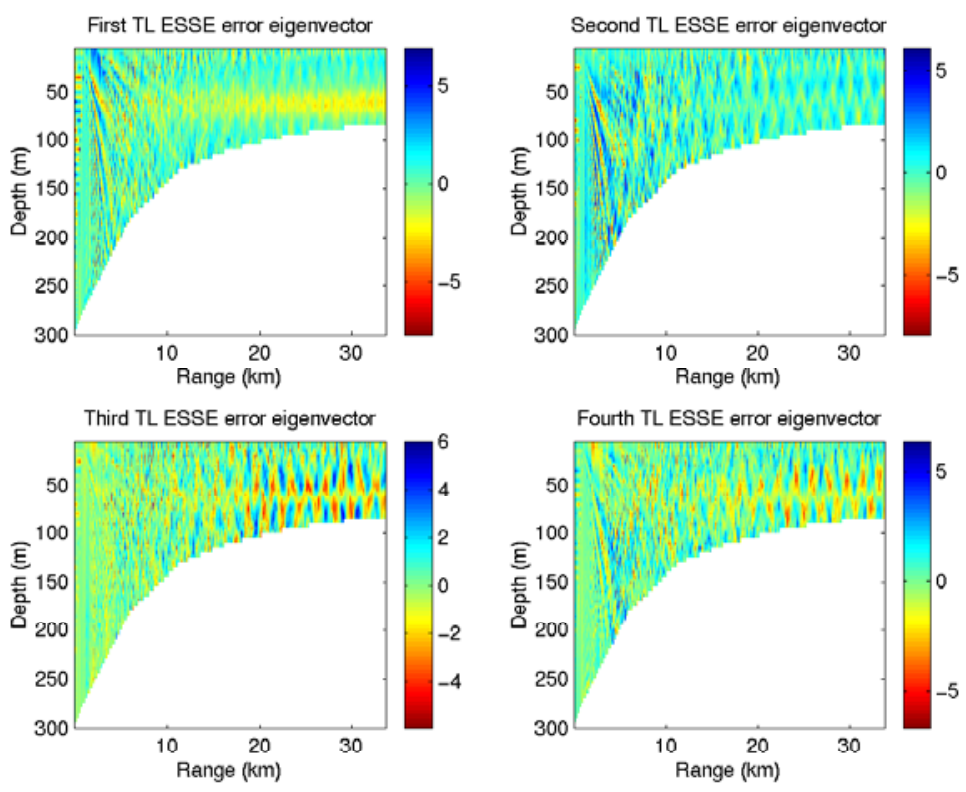

Figure 4. First four ESSE error eigenvectors for TL along section 1.

Figures 5 and 6 illustrate the data assimilation in the predicted error subspace (Figs. 34). The simulated true TL, a priori (i.e. the mean) TL, a posteriori TL and the TL realization closest to the a posteriori TL are shown on Fig. 5. Even though the true TL (Fig. 5a) is challenging to retrieve (TL of high-order modal interactions) and the sub-sampled data are limited, the a posteriori TL (Fig. 5c) is substantially closer to the true TL than the mean TL (Fig. 5b). From the ensemble of 79 TLs, one can select for best estimate the TL the closest (in some metric sense, here the RMS measure over the $r, z$ grid) to the a posteriori TL. This realization (Fig. 5d) is even a bit closer to the true TL than the a posteriori TL.

The differences between the a priori and true TLs, and between the a posteriori and true TLs, are shown on Figs. 6a-b. The a posteriori residuals (Fig. 6b) are much smaller than the a priori ones (Fig. 6a) at most locations, except above the thermocline near the surface on the shelf. This is due to the refractive effects of the thermocline (data are below 
COUPLED PHYSICAL-ACOUSTICAL DATA ASSIMILATION
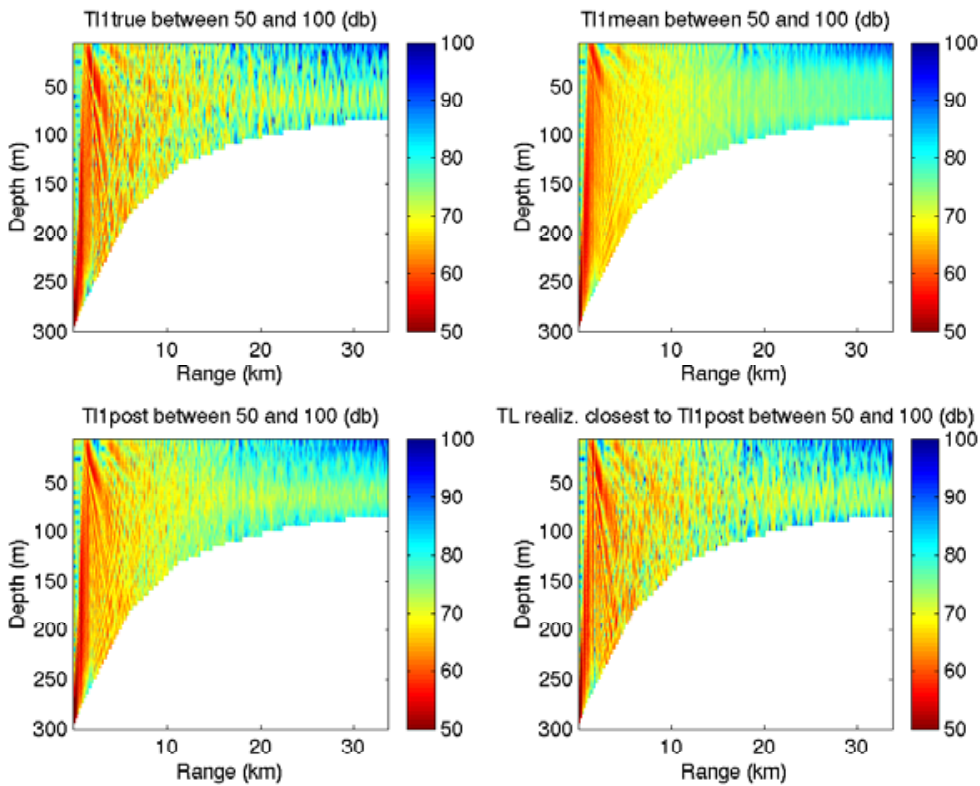

Figure 5. "True" TL, a priori TL, a posteriori TL and TL realization closest to a posteriori TL.

at $70 \mathrm{~m}$ ) and to the error subspace size (79) which is, based on convergence criteria, too small for accurate correlations everywhere in the large acoustic state $\left(\sim 410^{5}\right)$. With ESSE, error covariances are also estimated: the diagonals of the a priori and a posteriori error
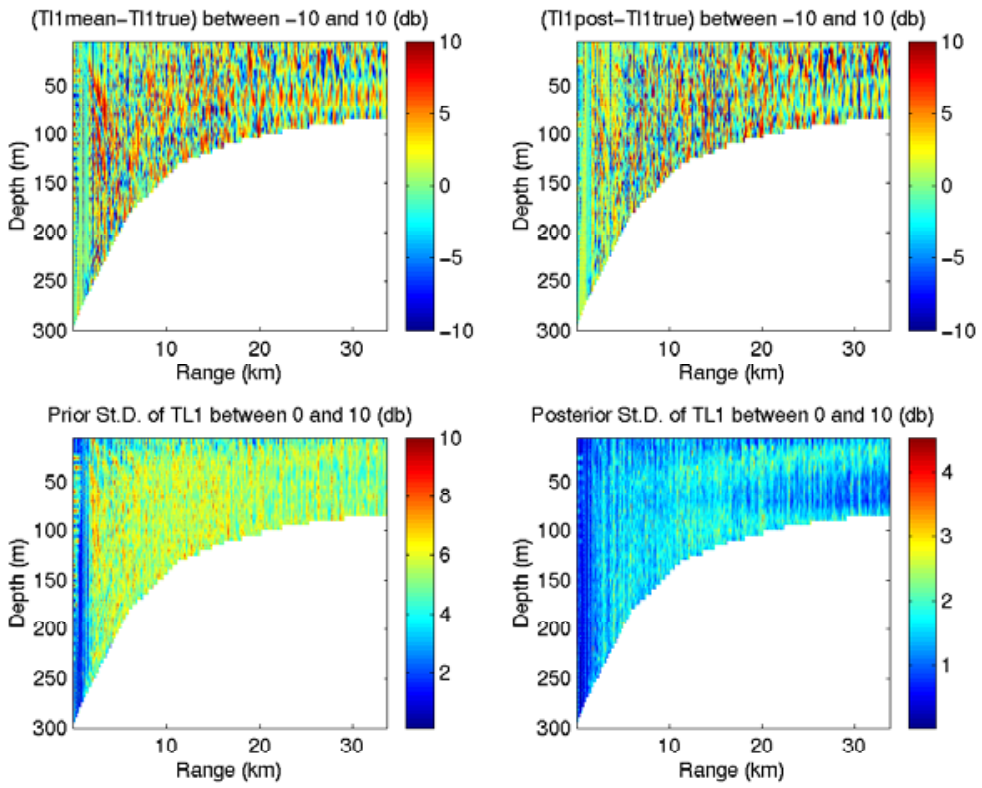

Figure 6. A posteriori residuals and a posteriori error St.Dv. for TL along section 1. 


\section{P.F.J. LERMUSIAUX AND C.S. CHIU}

covariances are illustrated on Fig. 6c-d. Overall, these standard deviations agree with the averages of the residuals (note that their accuracy increases with the subspace size). In particular, the expected error along the simulated towed-array at $70 \mathrm{~m}$ has been reduced.

\section{Conclusions}

Coupled four-dimensional data assimilation for physical-acoustical field estimates was carried successfully via Error Subspace Statistical Estimation in the context of an identical twin experiment. Physical uncertainties were transferred to acoustical uncertainties and the dominant acoustical error statistics were decomposed and their properties examined. Results are encouraging and such coupled four-dimensional data assimilations have the potential to provide significant advances in physical and acoustical ocean science.

\section{Acknowledgements}

We thank Prof. A.R. Robinson and ONR for support under grants N00014-00-1-0771 and N0001402WR20213. PFJL thanks P.J. Haley and W.G. Leslie for software and data expertise, and P. Elisseeff and H. Schmidt. This is a contribution of the UNITES team.

\section{References}

1. Chiu, C.-S., Downslope modal energy conversion, J. Ac. Soc. Am., 95(3), 1654-1657, (1994).

2. Chiu, C.-S., J.H. Miller, W.W. Denner, and J.F. Lynch, A Three-Dimensional, Broadband, Coupled Normal-Mode Sound Propagation Modeling Approach, in Full Field Inversion Methods in Ocean and Seismic Acoustics (O. Diachok, A. Caiti, P. Gerstoft and H. Schmidt editors), Kluwer Academic Publishers, 57-62 (1995).

3. Chiu, C.-S., J.H. Miller and J.F. Lynch, Forward coupled-mode propagation modeling for coastal acoustic tomography, J. Acoust. Soc. Am., 99(2), 793-802, (1996).

4. Lermusiaux, P.F.J. Data assimilation via Error Subspace Statistical Estimation. Part II: Middle Atlantic Bight shelfbreak front simulations and ESSE validation. Month. Weather Rev., 127(7), 1408-1432. (1999a).

5. Lermusiaux, P.F.J. Estimation and study of mesoscale variability in the Strait of Sicily. Dyn. of Atmos. Oceans, 29, 255-303. (1999b).

6. Lermusiaux P.F.J. and A.R. Robinson. Data assimilation via Error Subspace Statistical Estimation. Part I: Theory and schemes. Month. Weather Rev., 127(7), 1385-1407. (1999)

7. Lermusiaux P.F.J., D.G.M. Anderson and C.J. Lozano. On the mapping of multivariate geophysical fields: error and variability subspace estimates. Q.J.R. Meteorol. Soc., April B, 1387-1430. (2000).

8. Lermusiaux P.F.J., C.-S. Chiu and A.R. Robinson. Modeling uncertainties in the prediction of the acoustic wavefield in a shelfbreak environment. Proc. of the 5th International conference on theoretical and computational acoustics, May 21-25, 2001. Beijing, China. (2002)

9. Lynch, J.F., G.G. Gawarkiewicz, C.-S. Chiu, R. Pickart, J.H. Miller, K.B. Smith, A. Robinson, K. Brink, R. Beardsley, B. Sperry, and G. Potty. Shelfbreak PRIMER - An integrated acoustic and oceanographic field study in the mid-Atlantic Bight, in Shallow-Water Acoustics (R. Zhang and J. Zhou Editors), China Ocean Press, 205-212, (1997).

10. Robinson, A.R. Physical processes, field estimation and an approach to interdisciplinary ocean modeling. Earth-Science Rev., 40, 3-54. (1996)

11. Robinson A.R. and P.F.J. Lermusiaux. Data Assimilation in Models. Encyclopedia of Ocean Sciences, 623-634, Academic Press Ltd., London. (2001). 